\title{
Alternating RF/DC Isolations for Quantitation with Coeluting Internal Standards in Gas Chromatography/Ion Trap Mass Spectrometry
}

\author{
Brent L. Kleintop and Richard A. Yost \\ Department of Chemistry, University of Florida, Gainesville, Florida, USA \\ Craig R. Abolin \\ Sandoz Research Institute, East Hanover, New Jersey, USA
}

A new ion trap scan function for gas chromatography/mass spectrometry (GC/MS) quantitation is described that employs alternating mass-selective storage (rf/dc isolation) of ions from an analyte and its coeluting isotopically labeled internal standard. This scan includes two separate ionization/isolation/mass analysis sequences within the same scan function, each optimized for either the analyte or the internal standard. This results in alternating between analyzing the analyte and the internal standard during their coelution. The method is conceptually similar to using two different scan functions to analyze either the analyte or the internal standard in alternating scans; however, it is much faster because it eliminates the slow procedure of continuously downloading alternating scan functions from disk. This allows more data points to be obtained over a GC peak, resulting in more reproducible GC peak profiles as well as better sensitivity and precision. Results of calibration curves spanning four orders of magnitude ( $0.5 \mathrm{pg}$ to $5 \mathrm{ng}$ injected on column) obtained by using this method give excellent linear correlations $\left(r^{2}>0.9990\right)$ and precision (relative standard deviations of triplicate injections <10\%). (J Am Soc Mass Spectrom 1992, 3, 85-88)

$\mathrm{T}$ The quadrupole ion trap is an extremely sensitive and versatile mass spectrometer, largely owing to its ability to store ions prior to mass analysis. Indeed, good-quality, full-scan spectra of $<10 \mathrm{pg}$ of analyte are commonly obtained by gas chromatography/ion trap mass spectrometry (GC/ITMS) [1]. The instrumentation and operation of the ITMS have been described in several articles [2-4], and a recent book reviews the fundamentals and applications of the quadrupole ion trap [5].

A major disadvantage of the ion trap is its susceptibility to space charge effects that cause loss of sensitivity and mass resolution, and degradation of spectral quality. This has resulted in limited applications of this sensitive mass spectrometer to quantitative GC/MS analyses employing isotopically labeled internal standards (IS). An analyte and its isotopically labeled IS typically coelute, producing space charge conditions that limit the dynamic range of the instrument. Although feedback control of the ionization time via automatic gain control (AGC) has been shown to reduce dramatically the effects of space charge [6]

Address reprint requests to Richard A. Yost, Department of Chemistry, University of Florida, Gainesville, FL 32611-2046. and increase the dynamic range [1] as individual compounds are introduced into the trap, problems may occur when two compounds coelute. The AGC software selects optimum ionization times for the more abundant component, which may result in insufficient sensitivity and nonlinear response for the minor component.

The use of mass-selective storage ( $\mathrm{rf} / \mathrm{dc}$ isolation) to reduce the effects of space charge has been applied to quantitative analyses employing isotopically labeled internal standards $[7,8]$. The steps of the $\mathbf{r f} / \mathrm{dc}$ scan function have been described elsewhere [9]. Space charge was reduced by isolating a single massto-charge ratio corresponding to an ion characteristic of the analyte or the isotopically labeled IS in alternate scans during their coelution. These studies used an additional FORTH program to alternate between two different scan functions, each optimized for either the analyte or the IS. The scan functions are repeatedly loaded from the personal computer (PC) hard drive into the ITMS firmware, which is a relatively slow process. The ability to obtain only about one to two scans per second resulted in poor averaging and GC peak shape definition.

Recently, we have developed a new method of 
using alternating $\mathrm{rf} / \mathrm{dc}$ isolations of multiple ions that is used for quantitative analysis with isotopically labeled IS. A condensed version of the scan function is shown in Table 1. The method combines two $\mathrm{rf} / \mathrm{dc}$ isolation scan functions. Both contain separate ionization, isolation, and mass analysis (data acquisition) sequences optimized for either the analyte or the IS. Because both acquisitions are contained within one scan function, the ITMS software sums the data acquired from both "sub"-scans to create a single scan. Because of this, it is important that the mass analysis ranges of each subscan do not overlap. This also prevents any problems resulting from incomplete $\mathrm{rf} / \mathrm{dc}$ isolation of one component from the other because data are acquired with unit mass resolution for only one component in each subscan.

This new method provides a rapid means of alternating between $\mathrm{rf} / \mathrm{dc}$ isolating/analyzing an analyte and its isotopically labeled IS. The entire scan function is loaded from the PC hard drive into the ITMS firmware only once, at the beginning of the GC/MS analysis. This eliminates the time-consuming process of repeatedly accessing and loading the scan function into the firmware between every scan, and results in performing the same task more rapidly than in previous studies that utilized an additional FORTH program to control alternating between two scan functions. Results of regression analyses applied to analyses using this scan method on pure standards spanning four orders of magnitude are reported.

\section{Experimental}

Experiments were performed on a Finnigan-MAT (San Jose, CA) ion trap mass spectrometer interfaced to a Varian Associates (Walnut Creek, CA) model 3300 gas chromatograph. The spectrometer was maintained at $100^{\circ} \mathrm{C}$ for all experiments. Electron ionization was accomplished at an if voltage level of $\mathbf{4 5} \mathrm{u}$ and spectra

Table 1. Condensed listing of the ITMS scan function used in this study, including the component analyzed during each segment and the time required for each table, as well as total time for the entire scan function

\begin{tabular}{|c|c|c|}
\hline Component analyzed & Scan table(s) & Time (ms) \\
\hline $\begin{array}{c}\text { Analyte } \\
(\mathrm{m} / 2 \mathrm{163})\end{array}$ & $\left\{\begin{array}{l}\text { Trigger } \\
\text { First ionization pulse } \\
\text { First } r / d c \text { isolation } \\
\text { First data acquisition ramp } \\
\langle 161 \rightarrow 165 u) \\
\text { rf shut down }\end{array}\right.$ & $\begin{array}{l}0.15 \\
0.50 \\
2.70 \\
0.72 \\
0.10\end{array}$ \\
\hline $\begin{array}{l}\text { Internal standard } \\
(\mathrm{m} / \mathrm{z} 167)\end{array}$ & $\left\{\begin{array}{l}\text { Second ionization pulse } \\
\text { Second } r / d c \text { isolation } \\
\text { Second data acquisition ramp } \\
(165 \rightarrow 169 u) \\
\text { rf shut down }\end{array}\right.$ & $\begin{array}{l}1.00 \\
2.70 \\
0.72 \\
\frac{0.10}{8.69}\end{array}$ \\
\hline
\end{tabular}

were acquired over the scan range indicated in the text with an electron multiplier setting of $1400 \mathrm{~V}$ and a single conversion dynode operated at $-4 \mathrm{kV}$. The helium carrier gas of the gas chromatograph also serves as the buffer gas inside the ion trap; typical uncorrected manifold pressures were $\sim 3.5 \times 10^{-5}$ torr as measured by a Bayard-Alpert ionization gauge (Granville-Phillips, Boulder, CO) mounted on the vacuum chamber. Axial modulation was employed to reduce further the effects of space charge. Ionization times for the analyte and IS were 500 and $1000 \mu \mathrm{s}$, respectively, in all cases. Three microscans were averaged on the spectrometer and returned to the PC data system as a single scan. The PC data system consisted of an IBM PC/AT 286 operating at $8 \mathrm{MHz}$. Scan function times were measured on an oscilloscope (model 5440, Tektronix, Inc., Beaverton, OR).

Gas chromatography was carried out on an 11 $\mathrm{m} \times 0.25 \mathrm{~mm}$ i.d. DB-5 column (J \& W Scientific, Folsom, CA) with an $0.25-\mu \mathrm{m}$ film thickness. Helium was used as a carrier gas and adjusted to give a flow rate of $-1 \mathrm{~mL} / \mathrm{min}$. One-microliter injections were performed manually in split mode using a measured split ratio of 100:1. The chromatograph was held at $110^{\circ} \mathrm{C}$ for $30 \mathrm{~s}$ and then ramped to $180^{\circ} \mathrm{C}$ at a rate of $50^{\circ} \mathrm{C} / \mathrm{min}$. A homemade, resistively heated transfer line probe operated at $220^{\circ} \mathrm{C}$ was employed to interface the chromatograph to the spectrometer. The chromatography performed resulted in a baseline peakwidth of $-1 \mathrm{~s}$ at a retention time of just over 1 min.

The analyte and deuterated IS (shown in Figure 1) were provided by the Sandoz Research Institute (East Hanover, NJ). Isotopic labeling involved replacement of the two $\mathrm{CH}_{2}$ groups on the five-membered ring with $\mathrm{CD}_{2}$ groups, as indicated by asterisks in the frgure. Also shown is loss of the $R_{1}$ group upon electron ionization to form the fragment ions monitored, $163^{+}$and $167^{+}$for the analyte and IS, respectively. Samples were dissolved in methanol and serial dilutions were performed to produce standards with

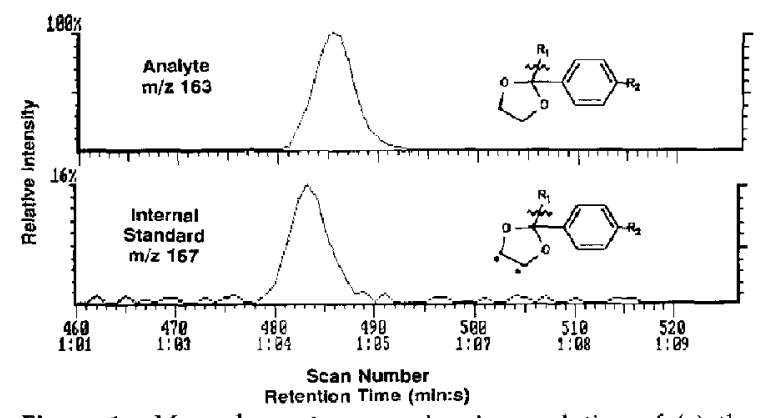

Figure 1. Mass chromatograms showing coelution of (a) the analyte (100 pg) and (b) the isotopically labeled IS (10 pg). Also shown are partial structures of both components as well as fragmentation of $R_{1}$ to form the fragment ions monitored. Asterisks indicate sites for isotopic labeling of IS. 
analyte concentrations ranging from $50 \mathrm{pg} / \mu \mathrm{L}$ to 500 $\mathrm{ng} / \mu \mathrm{L}$. The concentration of deuterated IS was 1000 $\mathrm{pg} / \mu \mathrm{L}$ in all cases. This resulted in $0.5 \mathrm{pg}$ to $5 \mathrm{ng}$ of analyte and $10 \mathrm{pg}$ of IS being injected onto the chromatograph column.

One modification to the standard spectrometer software was necessary for these studies. Present ITMS software stops data acquisition $4 \mathrm{u}$ below the end (high) mass specified by the user in the data acquisition table of a scan function due to a bug in the software. Modification of the ITMS software allowed acquisition over the entire mass range specified in the data acquisition table of the scan function. This modification is not necessary for analyses employing an analyte and IS that are separated by more than $4 \mathrm{u}$; however, compiled copies of the modified user programs used in these studies are available to ITMS users from the corresponding author upon request.

\section{Results and Discussion}

Times measured on an oscilloscope showed that to acquire and average three microscans for both components requires $-105 \mathrm{~ms}$. Timing traces for the scan function described here and in the previously used method $[7,8]$ are shown in Figure 2. These traces are shown for one microscan/scan (no scan averaging) for clarity. Also shown is the time required to analyze and acquire data for both components using each method. These traces clearly show that the method a) New scan method

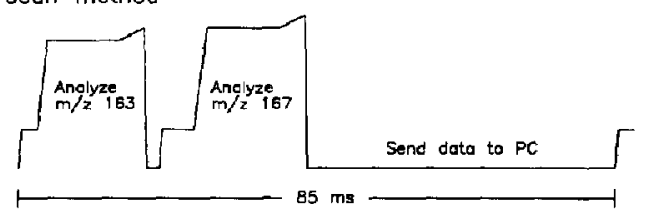

b) Previously used method

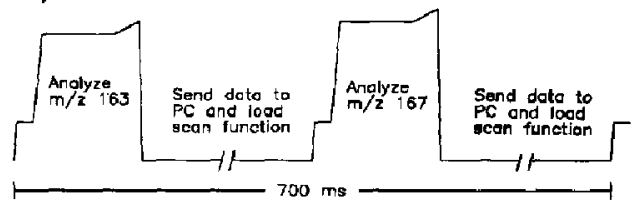

Figure 2. Scan function timing diagrams showing if amplitude versus time (not to scale) indicating the time required to analyze the analyte and IS with (a) the new and (b) previously used [7, 8] scan methods. Trace a corresponds to the scan functions listed in Table 1.

described here is more than eight times faster than the previously used method. The increased acquisition rate allows acquisition of more data points over a GC peak, which results in more reproducible peak shapes and areas for quantitation. Figure 3 shows mass chromatograms obtained for triplicate injections of $50 \mathrm{pg}$ of analyte using both the scan function described here and the previously used method. The peaks in the chromatograms obtained by using the

\section{a) New scan method}
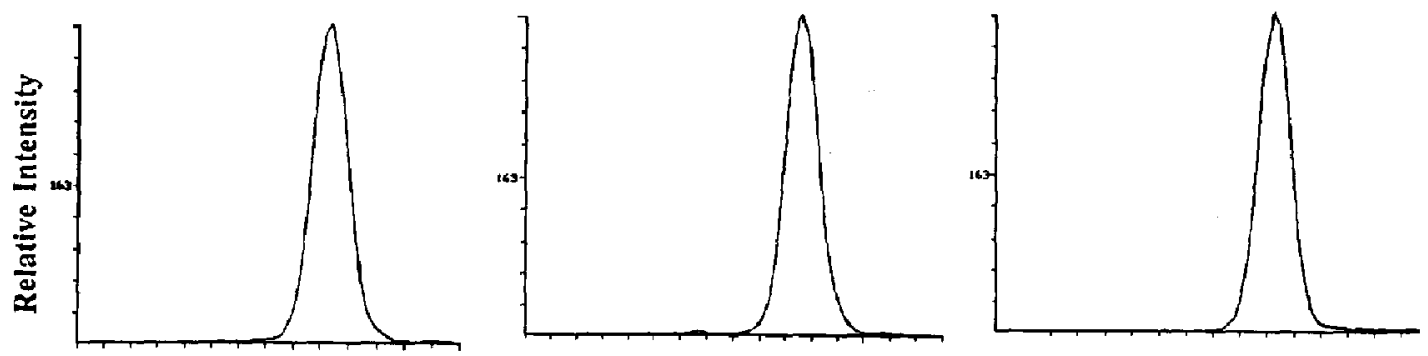

b) Previously used method
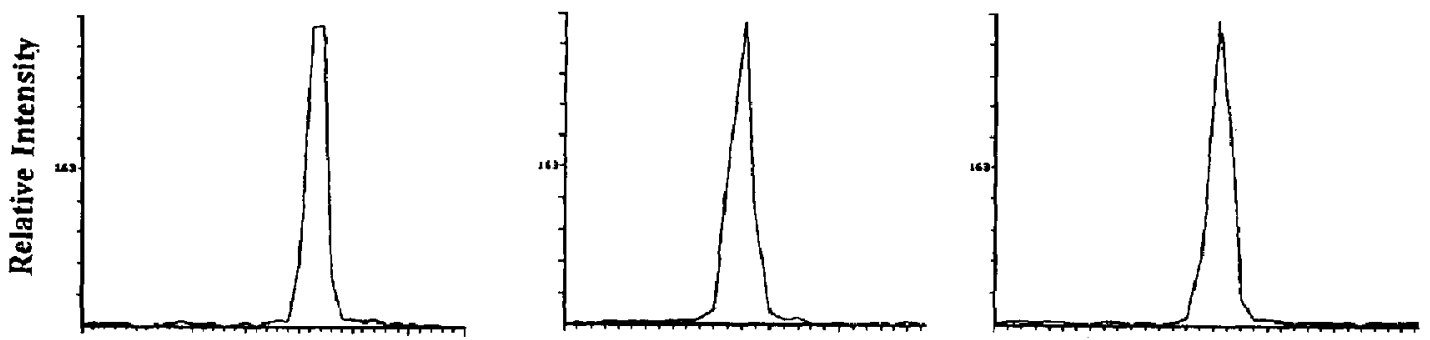

Retention Time

Figure 3. Mass chromatograms ( $m / z$ 163) of analyte (50 pg) obtained from triplicate injections using (a) the new and (b) previously used scan methods. 


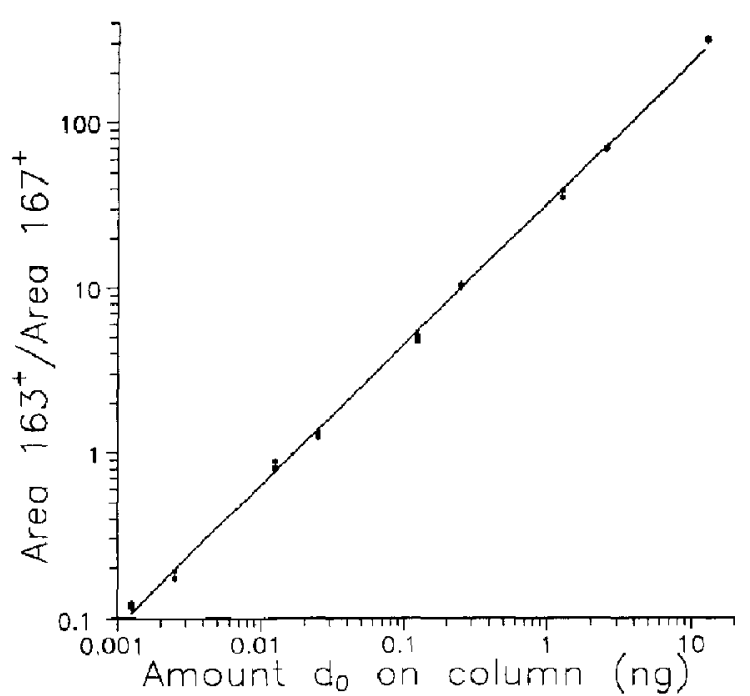

Figure 4. Instrument calibration curve (log-log) for triplicate injections spanning four orders of magnitude for compound studied using scan function described in text.

new scan method show excellent peak shape reproducibility, whereas those from the latter are irreproducibly skewed or shaved off.

Figure 1 presents typical mass chromatograms showing coelution of the analyte and the IS. A typical calibration curve in log-log format spanning four orders of magnitude shows excellent linearity, as shown in Figure 4. Linear regression revealed correlation coefficient values $\left(r^{2}\right)>0.9990$ in all studies performed. The calibration curves and linear regression included all data points from triplicate injections. Precisions of triplicate injection was better than $10 \%$ RSD over the entire range of concentrations studied. Slopes $<1$ (typically 0.7 ) resulted from differences in the ionization times chosen for both components, as well as possible differences in the isolation efficiencies of the ions.

\section{Conclusions}

We have demonstrated a new method of rapidly performing alternating $\mathrm{rf} / \mathrm{dc}$ isolation scans on the ion trap mass spectrometer. When applied to GC/ITMS analyses employing isotopically labeled IS, this method shows good linearity over four orders of magnitude. The sensitivity, linearity, and reproducibility are due to the ability to alternate between analyzing analyte and IS ions rapidly enough to obtain an adequate number of data points (eight to nine) over a GC peak -1 s wide.

We are currently evaluating this scan method over a wider concentration range. We also plan to evaluate the method with splitless injection for improved absolute detection limits and for extracted plasma samples.

\section{Acknowledgments}

The authors acknowledge partial funding for this project from the Sandoz Research Institute. We also thank Mr. Nathan Yates for his help with modifying the ITMS software and Dr. Jodie Johnson for fruitful discussion of the results of these studies.

\section{References}

1. Yost, R. A.; McClennen, W.; Snyder, A. P. Presented at the 35th ASMS Conference on Mass Spectrometry and Allied Topics, Denver, CO, May 24-29, 1987, p. 789.

2. Louris, J. N.; Brodbelt, J. S.; Cooks, R. G. Int. J. Mass Spectrom. Ion Processes 1987, 75, 345-352.

3. Louris, J. N.; Cooks, R. G.; Syka, J. E. P.; Kelley, P. E.; Stafford, G. C.; Todd, J. F. J. Anal. Chem. 1987, 59, $1677-1685$

4. Mcluckey, S. A.; Glish, G. L.; Kelley, P. E. Anal. Chem. 1987, 59, 1670-1674.

5. March, R. E.; Hughes, R. J. Quadrupole Storage Mass Spectrometry; Wiley: New York, 1989.

6. Stafford, G. C.; Taylor, D. M.; Bradshaw, S. E.; Syka, J. E. P.; Uhrich, M. Presented at the 35th ASMS Conference on Mass Spectrometry and Allied Topics, Denver, CO, May 24-29, 1987, p. 775 .

7. Strife, R. J.; Simms, J. R.; Lacey, M. P. J. Am. Soc. Mass Spectrom. 1990, 1, 265-271.

8. Kleintop, B. L.; Johnson, J. V.; Yost, R. A.; Abolin, C. A. Presented at the 38th ASMS Conference on Mass Spectrometry and Allied Topics, Tucson, AZ, June 3-8, 1990, p. 872.

9. Strife, R. J.; Kelley, P. E.; Weber-Grabau, M. Rapid Commun. Mass Spectrom. 1988, 2, 105-109. 